\title{
Selective degradation of the IKB kinase (IKK) by autophagy
}

\author{
Dangsheng $\mathrm{Li}^{1}$ \\ ${ }^{1}$ Cell Research, Shanghai Institutes for Biological Sciences, Chinese Academy of Sciences, 319 Yueyang Road, Shanghai 200031, \\ China
}

Cell Research (2006) 16:855-856. doi:10.1038/sj.cr.7310110; published online 16 November 2006

Proteasome-mediated degradation and autophagy are the two major pathways mediating the turnover of cellular proteins. The proteasomal pathway is known to be a highly specific and regulated process mediating the degradation of short-lived proteins such as many important factors involved in cellular signaling. In contrast, it is generally thought that autophagy is rather nonselective as it is responsible for the bulk degradation of long-lived proteins and organelles. Challenging this general view, in this issue of Cell Research, Qing et al. report that selective degradation of the IKB kinase (IKK) triggered by the loss of Hsp90 function is mediated by autophagy [1].

IKK phosphorylates I $\mathrm{KB}$, marking it for subsequent proteasomal degradation. It is thus a critical regulator of the NF$\kappa \mathrm{B}$ pathway, which holds prominent roles in inflammation and cancer. Hsp90 is a well-known molecular chaperone that functions to assist the proper folding of many client proteins. Inhibition of $\mathrm{Hsp} 90$ function often leads to degradation of its clients presumably as a result of their improper folding; and degradation of these clients has all been known to be mediated by the proteasomal pathway until the study by Qing et al [1]. Led by the recent finding that IKK is a client of Hsp90 [2], the authors started by examining the protein levels of all three IKK subunits in a number of cell lines treated with geldanamycin (GA), a specific inhibitor of Hsp90. Perhaps not surprisingly, they found that in all cases, GA treatment induced a significant reduction in IKK protein levels. Additional experiments indicated that reduced IKK expression is due to enhanced degradation. While all these results seemed to be expected, a surprise emerged when the authors further investigated the underlying mechanism and found that IKK degradation induced by GA did not appear to involve the proteasomal pathway. This was supported by two main pieces of evidence. First, the authors employed a mutant cell line harboring a temperature-sensitive mutation in the E1 ubiquitin-activating enzyme that is critical for the ubiquitination reaction. Shifting these cells to a higher temperature inactivates the E1 and abolishes ubiquitination; and the authors found that under such conditions known substrates of the ubiquitin/proteasomal pathway were stabilized. In contrast, inactivation of E1 did not affect GA-induced degradation of IKK. Second, the proteasome inhibitor MG132 also failed to block GA-induced IKK degradation.

To shed more light on the mechanism of IKK degradation triggered by the loss of Hsp90 function, Qing et al. probed the potential role of autophagy as it is the other main system for protein turnovers in cells. They "interfered" with cellular autophagy by treating cells with a chemical inhibitor of autophagy or by using cells that genetically lack an essential component of the autophagy machinery; and interestingly, they found in both cases GA-induced degradation of IKK was significantly inhibited. As an important control, the authors showed that interfering with autophagy had no effect on GA-induced degradation of Akt, another client of Hsp90 that is known to be degraded by the proteasomal pathway upon inhibition of Hsp90 function. Taken together, the results by Qing et al. clearly indicate that the selective degradation of IKK in the absence of Hsp90 function is mediated by the autophagy pathway, revealing a novel link between Hsp90 and autophagy. The study by Qing et al. also suggests that under certain circumstances cells can employ the autophagy pathway to achieve specific degradation of a signaling molecule, and raises tantalizing questions regarding the mechanism(s) by which the autophagy pathway/machinery selects its target substrate (in this case, IKK). Given that the Hsp90 inhibitor GA is an anti-tumor agent and given the prominent role of the NF- $\mathrm{BB}$ pathway in cancer and inflammation, further understanding of the underlying mechanism(s) may also have therapeutic implications. 


\section{References}

1 Qing G, Yan P, Xiao G. Hsp90 inhibition results in autophagy-mediated proteasome-independent degradation of IкB kinase (IKK). Cell Res 2006; 16:895-901.

2 Chen G, Cao P, Goeddel DV. TNF-induced recruitment and activation of the IKK complex require Cdc37 and Hsp90. Mol Cell 2002; 9:401-410. 